\title{
THE STRUCTURE AND IDEAL THEORY OF THE PREDUAL OF A BANACH LATTICE
}

BY

E. B. DAVIES

1. Introduction. If $V$ is an ordered Banach space over the real field, then the Banach dual $V^{*}$ has a natural induced partial ordering. In Theorems 3.1 and 3.2 we present necessary and sufficient conditions for $V^{*}$ to be a Banach lattice, extending partial results obtained by Choquet [5] and Andô [1]. The theorems include as special cases the characterisations of the predual of an $M$-space, [8], [14], and the predual of an $L$-space, [6], called a simplex space in [11]. We show how the theory is a natural generalisation of Choquet simplex theory.

If $V^{*}$ is a Banach lattice, we study the set of closed ideals of $V$. For the special case of simplex spaces, the results provide direct proofs of theorems in [11].

2. Basic theorems on ordered Banach spaces. An ordered normed space $V$ over the real field is defined as a normed vector space $V$ with a closed cone $C$ which is proper in the sense that $C \cap(-C)=\{0\}$ and with the partial ordering given by saying that $x \leqq y$ if and only if $y-x \in C$. $V$ is said to be positively generated if $C-C=V$ and to have a monotone norm if $0 \leqq x \leqq y$ implies $\|x\| \leqq\|y\| . V$ is said to be regular if it has the properties

(i) if $x, y \in V$ and $-x \leqq y \leqq x$ then $\|y\| \leqq\|x\|$;

(ii) if $x \in V$ and $\varepsilon>0$ then there is some $y \in V$ with $y \geqq x,-x$ and $\|y\|<\|x\|+\varepsilon$.

A normed lattice is a regular ordered normed space which is a lattice under its partial ordering, and a Banach lattice is a complete normed lattice. Banach lattices are discussed in many places, for example [7], [12], and we follow the standard terminology. A partially ordered vector space $V$ is said to have the Riesz separation property if when $a, b \leqq c, d \in V$ we can find some $x \in V$ with

$$
a, b \leqq x \leqq c, d .
$$

For alternative formulations of this condition see [1], [9].

Lemma 2.1. Let $V$ be an ordered positively generated Banach space with a monotone norm $\|\quad\|$, and define

$$
\|x\|_{1}=\inf \{\|y\|: x,-x \leqq y \in V\}
$$

for all $x \in V$. Then \|\|$_{1}$ is a regular norm on $V$ which is equivalent to \|\| , and for any $0 \leqq x \in V$ we have $\|x\|_{1}=\|x\|$.

For a proof of this see [16].

Received by the editors February 17, 1967. 
Let $V$ be a positively generated ordered Banach space with Banach dual $V^{*}$. The set $C^{*}$ of continuous positive functionals is a weak*-closed proper cone in $V^{*}$. By the Hahn-Banach theorem if $x \in V$ then $x \geqq 0$ if and only if $(x, \phi) \geqq 0$ for all $\phi \in C^{*}$. If $V$ is a positively generated ordered Banach space with a monotone norm then, by [16], every positive functional on $V$ is continuous; also if $0 \leqq x \in V$ then

$$
\|x\|=\max \left\{(x, \phi): \phi \in C^{*} \cdot\|\phi\| \leqq 1\right\} .
$$

This follows from the fact that $-x$ is not in the open convex set

$$
\{v \in V: \exists c \in C \cdot\|v-c\|<\|x\|\}
$$

by the use of the Hahn-Banach theorem. If $V$ is a regular ordered Banach space then for any $0 \leqq \phi \in V^{*}$ we have

$$
\|\phi\|=\sup \{(x, \phi): x \in C \cdot\|x\| \leqq 1\} .
$$

LEMMA 2.2. If $V$ is a regular ordered normed space then for any $\phi, \psi \in V^{*}$ with $-\phi \leqq \psi \leqq \phi$ we have $\|\phi\| \geqq\|\psi\|$.

For let $x \in V$ and $\|x\|<1$. If $z \geqq x, 0$ then

$$
\begin{aligned}
(\psi, x) & =(\psi, z)+(\psi, x-z) \\
& \leqq(\phi, z)+(\phi, z-x) \\
& =(\phi, 2 z-x) .
\end{aligned}
$$

If $y \in V$ satisfies $y \geqq x,-x$ and $\|y\|<1$ and we put $z=(x+y) / 2$ then we obtain

$$
(\psi, x) \leqq\|\phi\|\|y\|<\|\phi\| \text {. }
$$

The result now follows immediately.

Very much more information can be obtained about $V^{*}$, but we first need to consider a special case.

LEMMA 2.3. Let $V$ be a regular ordered normed space with the Riesz separation property. Then $V^{*}$ is a Banach lattice.

Let $\phi \in V^{*}$ and $x \in V, x \geqq 0$. Then we define

$$
\left(\phi^{+}, x\right)=\sup \{(\phi, y): 0 \leqq y \leqq x\} .
$$

It is easy to see that $\phi^{+}$can be uniquely extended to a positive linear functional on $V$, and that $\phi^{+}$is continuous with $\left\|\phi^{+}\right\| \leqq\|\phi\|$. Clearly, $V^{*}$ is a lattice with $\phi \vee 0$ $=\phi^{+}$. Define $|\phi|=2 \phi^{+}-\phi$. For any $y \in V, y \geqq 0$ we can find some $a \in V$ with $0 \leqq a \leqq y$ and

which gives

$$
(\phi, a) \leqq\left(\phi^{+}, y\right) \leqq(\phi, a)+\varepsilon / 2
$$

As $-y \leqq 2 a-y \leqq y$ so

$$
|(|\phi|, y)-(\phi, 2 a-y)|<\varepsilon .
$$

$$
|(\phi, 2 a-y)| \leqq\|\phi\|\|y\|
$$

from which we conclude that $\||\phi|\| \leqq\|\phi\|$. The result now follows immediately. 
Let $V$ be an ordered positively generated Banach space and define:

$$
\Delta=C^{*} \cap\left\{\phi \in V^{*}:\|\phi\| \leqq 1\right\} .
$$

$\Delta$ is a weak*-compact convex set with $0 \in \Delta$ and there is a natural map $\lambda: V$ $\rightarrow A_{0}(\Delta)$, the space of all continuous affine functionals $\phi$ on $\Delta$ such that $\phi(0)=0$. By our previous remarks $\lambda$ is an order isomorphism of $V$ into $A_{0}(\Delta)$, and if \|\|$_{u}$ denotes the supremum norm in $A_{0}(\Delta)$, we have for all $f \in V$

$$
\|\lambda f\|_{u} \leqq\|f\| \text {. }
$$

Identifying $V$ with $\lambda V$ we define $S$ as the cone of functions of the form $f_{1} \vee \cdots \vee f_{n}$ where $f_{r} \in V$. Then if $L=S-S, L$ is a vector lattice of continuous functions on $\Delta$. See [5]. For $\phi \in L$ define

$$
\|\phi\|=\inf \{\|\psi\|: \psi \in V \cdot \psi \geqq \phi,-\phi\} .
$$

Then, provided $V$ is positively generated and has a monotone norm, $L$ becomes a normed lattice and $V$ is canonically embedded as a Banach subspace of $L$.

LEMMA 2.4. If $V$ is a regular ordered Banach space then $V^{*}$ is also regular.

Let $\phi \in V^{*}$ and $\|\phi\| \leqq 1$. We can, by the Hahn-Banach theorem, extend $\phi$ to a functional $\xi \in L^{*}$ with $\|\xi\| \leqq 1$. As $L^{*}$ is a Banach lattice so by defining $\psi$ as the restriction to $V$ of $|\xi|$, we see that $\psi \geqq \phi,-\phi$ and $\|\psi\| \leqq 1$.

LEMMA 2.5. If $V$ is an ordered Banach space such that $V^{*}$ is regular then the map $\lambda: V \rightarrow A_{0}(\Delta)$ is a one-one onto order isomorphism such that for all $f \in V$

$$
\|\lambda f\|_{u} \leqq\|f\| \leqq 2\|\lambda f\|_{u}
$$

and for all $0 \leqq f \in V$,

$$
\|\lambda f\|_{u}=\|f\| .
$$

That $\|\lambda f\|_{u} \leqq\|f\|$ is clear. Suppose $f \in V$ and $|(\phi, f)|=\|f\|$ where $\|\phi\|=1$. Then for any $\varepsilon>0$ we find $\psi \in V^{*}$ with $\psi \geqq \phi,-\phi,\|\psi\|<1+\varepsilon$.

$$
|(\phi, f)|=|((\psi+\phi) / 2, f)-((\psi-\phi) / 2, f)|
$$

so that

$$
\max |((\psi \pm \phi) / 2, f)| \geqq \frac{1}{2}\|f\| .
$$

As $(\psi \pm \phi) / 2 \geqq 0$ and $\|(\psi \pm \phi) / 2\|<1+\varepsilon$ so

$$
\|f\| \leqq 2\|\lambda f\|_{u} .
$$

If $f \geqq 0$ then $((\psi \pm \phi) / 2, f) \geqq 0$ so that

$$
|(\phi, f)| \leqq \max |((\psi \pm \phi) / 2, f)|,
$$

from which we see that

$$
\|f\| \leqq\|\lambda f\|_{u}
$$


We see that $V$ maps onto a uniformly closed subspace of $A_{0}(\Delta)$ which separates the points of $\Delta$, and so must conclude by Lemma 4.3 of [13] that

$$
\lambda V=A_{0}(\Delta) .
$$

A partial converse to Lemma 2.4 is contained in the following

LEMMA 2.6. Let $V$ be an ordered Banach space such that $V^{*}$ has a proper positive dual cone $C^{*}$ and is regular. Then $V$ is positively generated and for any $x, y \in V$ with $-x \leqq y \leqq x$ we have $\|y\| \leqq\|x\|$.

For let $\psi \in V^{*}$ and $\|\psi\|<1$. Let $\phi \in V^{*}$ satisfy $\phi \geqq \psi,-\psi$ and $\|\phi\|<1$. Then

$$
\begin{aligned}
(\psi, y) & =((\psi+\phi) / 2, y)-((\phi-\psi) / 2, y) \\
& \leqq((\psi+\phi) / 2, x)+((\phi-\psi) / 2, x) \\
& =(\phi, x)<\|x\| .
\end{aligned}
$$

Thus $\|y\| \leqq\|x\|$. That $V$ is positively generated follows from [1] upon observing that $V^{*}$ is $\sigma-(0)$ complete.

We continue to suppose that $V$ is an ordered Banach space and that $V^{*}$ is regular. Following [5], we now define a conical measure $\mu$ as a positive functional in $L^{*}$. Such a functional defines by restriction to $V$ a point of $C^{*}$ called the barycentre of $\mu$. The set $P$ of conical measures $\mu$ with $\|\mu\| \leqq 1$ is compact in the $\sigma\left(L^{*}, L\right)$ topology. We define a closed partial ordering, $\prec$, on $P$ by

$$
\mu \prec \nu \quad \text { if } \quad(\mu, f) \leqq(\nu, f) \quad \text { for all } f \in S .
$$

We say $\mu \in P$ is a representing conical measure for $x \in \Delta$ if $f(x) \leqq(\mu, f)$ for all $f \in S$, and observe that every $x \in \Delta$ has at least one maximal representing conical measure.

If $x_{1}, \ldots, x_{n} \in \Delta$ and $x_{1}+\cdots+x_{n}=x \in \Delta$ then the functional

$$
f \sim f\left(x_{1}\right)+\cdots+f\left(x_{n}\right)
$$

is called a discrete conical measure, and is obviously a representing conical measure for $x$. We show that such conical measures are dense in $P$ in a very good sense.

LEMMA 2.7. Suppose $\mu \in P$ is a representing conical measure for $x \in \Delta$ and that $f_{r} \in S$ for $r=1, \ldots, n$. Then there is a discrete representing conical measure $\nu$ for $x$ such that $\mu\left(f_{r}\right)=\nu\left(f_{r}\right)$ for $r=1, \ldots, n$.

We can write the weak*-closed cone $C^{*} \subseteq V^{*}$ as a union of closed cones $D_{1}, \ldots$, $D_{m}$ such that $f_{r} \mid D_{s}$ are equal to the restrictions of functions in $A_{0}(\Delta)$. If $f \in L$ and $\geqq 0$, we define

$$
\left(\mu_{D_{s}}, f\right)=\inf \left\{(\mu, g): 0 \leqq g \in L \cdot g\left|D_{s}>f\right| D_{s}\right\} .
$$

Then $\mu_{D_{s}} \in P$ and $\mu \leqq \mu_{D_{1}}+\cdots+\mu_{D_{m}}$. Now we choose $\mu_{s} \in P$ such that $0 \leqq \mu_{s} \leqq \mu_{D_{s}}$ and $\mu=\mu_{1}+\cdots+\mu_{m}$. If $f \in L$ and $f \mid D_{s} \geqq 0$ then

$$
\left(f, \mu_{s}\right) \geqq\left(f \wedge 0, \mu_{s}\right) \geqq\left(f \wedge 0, \mu_{D_{s}}\right)=0 .
$$


Thus $\mu_{s}$ is determined by the value of $f$ on $D_{s}$ alone. The barycentre $x_{s}$ of $\mu_{s}$ is in $D_{s}$. Finally for $r=1, \ldots, n$

$$
\left(f_{r}, \mu\right)=\sum_{s=1}^{m}\left(f_{r}, \mu_{s}\right)=\sum_{s=1}^{m}\left(f_{r}\left(x_{s}\right)\right)
$$

Lemma 2.8. Suppose $f_{r} \in V$ and $f_{1} \vee \cdots \vee f_{n}=f \in S$. Then

$$
\max \left\{(f, \mu): \varepsilon_{x}<\mu\right\}=\max \left\{\sum_{r=1}^{n} f\left(x_{r}\right): x_{r} \in \Delta . \sum x_{r}=x\right\}
$$

and this quantity is called $\hat{f}(x)$. The map $x \rightarrow \hat{f}(x)$ is upper semicontinuous (u.s.c.).

The set

$$
\left\{\left\{x_{r} \in \Delta\right\}_{r=1}^{n}: \sum_{r=1}^{n} x_{r}=x \in \Delta\right\}
$$

is a closed subset of $\Delta^{n}$ and so by the continuity of the $f_{r}$ we see that the supremum of the right-hand side is always attained. The two sides are equal by Lemma 2.7. That $\hat{f}$ is upper semicontinuous follows from the equation

$$
\{x \in \Delta: \hat{f}(x) \geqq \alpha\}=\left\{x=\sum_{r=1}^{n} x_{r}: \sum_{r=1}^{n} f_{r}\left(x_{r}\right) \geqq \alpha\right\} .
$$

The following extension of the above lemma to semicontinuous functions will be important in our later discussion of closed ideals.

LEMMA 2.9. Let $f_{r}: \Delta \rightarrow[-\infty, \infty)$ be upper semicontinuous affine functionals with $f_{r}(0)=0$. Then for $f=f_{1} \vee \cdots \vee f_{n}$ and $x \in \Delta$ the supremum

$$
\sup \left\{\sum_{r=1}^{n} f\left(x_{r}\right): \sum_{r=1}^{n} x_{r}=x . x_{r} \in \Delta\right\}
$$

is always attained and is denoted $\hat{f}(x) . \hat{f}$ is an upper semicontinuous function with $\hat{f}(0)=0$ and $\hat{f}(\alpha . x)=\alpha \hat{f}(x)$ for all $x \in \Delta$.

The proof is as for Lemma 2.8.

\section{The main theorems.}

THEOREM 3.1. Let $V$ be an ordered Banach space, such that $V^{*}$ is regular. Then the following statements are equivalent:

(i) $V$ is regular and has the Riesz separation property;

(ii) $V^{*}$ is a lattice;

(iii) the map $f \sim \rightarrow \hat{f}(x)$ is linear for any $x \in \Delta$;

(iv) every $x \in \Delta$ has a unique maximal representing conical measure;

(v) the map $\sim \rightarrow \hat{f}(x)$ is linear for any $f \in S$.

As an immediate corollary we have 
Theorem 3.2. Let $V$ be an ordered Banach space. Then $V^{*}$ is a Banach lattice if and only if $V$ is regular and has the Riesz separation property.

We now prove Theorem 3.1. The implication (i) $\rightarrow$ (ii) was shown in Lemma 2.3. The implications (ii) $\rightarrow$ (iii) $\rightarrow$ (iv) $\rightarrow$ (v) can be found in [5]. The equivalence (i) $\leftrightarrow$ (ii) is closely related to a Theorem in [1], but is more specific. We now show (v) $\rightarrow$ (i).

Suppose $f, g, h \in V$. Then $(f \vee g)^{\wedge}$ is u.s.c. affine and so we can define $\left((f \vee g)^{\wedge} \vee h\right)^{\wedge}$ as in Lemma 2.9. It is easy to show that

$$
\left((f \vee g)^{\wedge} \vee h\right)^{\wedge}=(f \vee g \vee h)^{\wedge}
$$

and so is u.s.c. affine.

Next suppose that $f, g$ are u.s.c. affine on $\Delta$ and $f, g \leqq h$ where $h$ is affine. Then $(f \vee g)^{\wedge} \leqq h$ without any continuity assumptions for $h$.

For $f \in-S$ we define $f^{\vee}$ by $f^{\vee}=-(-f)^{\wedge}$. Now let $f_{1}, f_{2}, g_{1}, g_{2} \in V$ and $f_{1} \vee f_{2}$ $\leqq g_{1} \wedge g_{2}$. Then if $f=\left(f_{1} \vee f_{2}\right)^{\wedge}$ and $g=\left(g_{1} \wedge g_{2}\right)^{\vee}$ we have $f \leqq g$. We construct a sequence $h_{n} \in A_{0}(\Delta) \equiv V$ such that

$$
f-1 / 2^{n}<h_{n}<g+1 / 2^{n} .
$$

Suppose $h_{n}$ is given. Let $z \in \Delta$ and $z=x+y$ where $x, y \in \Delta$ and

$$
\left(f \vee h_{n}\right)^{\wedge}(z)=f(x)+h_{n}(y)<\left(h_{n}(z)+1 / 2^{n}\right) \wedge\left(g(z)+1 / 2^{n}\right) .
$$

Now $\left(f \vee h_{n}\right)^{\wedge}$ is u.s.c. affine and so by simple convexity arguments to be found in [3], we can find $k_{n} \in A(\Delta)$ with

$$
\left(f \vee h_{n}\right)^{\wedge}-1 / 2^{n+1}<k_{n}<h_{n} \wedge g+1 / 2^{n+1},
$$

and also satisfying $k_{n}(0)<0$.

Similarly we can find $1_{n} \in A(\Delta)$ with

$$
f \vee h_{n}-1 / 2^{n+1}<1_{n}<\left(h_{n} \wedge g\right)^{\vee}+1 / 2^{n+1},
$$

and also satisfying $h_{n}(0)>0$.

Putting $h_{n+1}=\lambda k_{n}+(1-\lambda) 1_{n}$ for a suitable $0<\lambda<1$ we obtain

$$
\left(f \vee h_{n}\right)-1 / 2^{n+1}<h_{n+1}<\left(h_{n} \wedge g\right)+1 / 2^{n+1},
$$

and $h_{n+1}(0)=0$.

Then

$$
\left\|h_{n}-h_{n+1}\right\|_{u}<1 / 2^{n+1},
$$

so $h_{n} \rightarrow h \in A_{0}(\Delta)$. It is then clear that

$$
f_{1} \vee f_{2} \leqq f \leqq h \leqq g \leqq g_{1} \wedge g_{2},
$$

so that $V$ has the Riesz separation property. 
We now show that $V$ is regular. Let $f \in A_{0}(\Delta)$ and $z \in \Delta$. Then we can find $x$, $y \in \Delta$ with $x+y=z$ and satisfying

$$
\begin{aligned}
\{f \vee(-f)\}^{\wedge}(z) & =f(x)-f(y) \\
& =f(x-y) .
\end{aligned}
$$

Now $-z \leqq x-y \leqq z$ so by the regularity of $V^{*}$

$$
\{f \vee(-f)\}^{\wedge}(z) \leqq\|f\| .
$$

Now suppose $\varepsilon>0$. We define $h_{1} \in A_{0}(\Delta)$ so that

$$
\{f \vee(-f)\}^{\wedge}-\varepsilon / 2<h_{1}<\|f\|+\varepsilon / 2
$$

Inductively we construct $h_{n} \in A_{0}(\Delta)$ with

$$
\{f \vee(-f)\}^{\wedge}-\varepsilon / 2^{n}<h_{n},
$$

and more exactly with

$$
\{f \vee(-f)\}^{\wedge} \vee h_{n}-\varepsilon / 2^{n+1}<h_{n+1}<h_{n}+\varepsilon / 2^{n+1} .
$$

This can be done by the same procedure as above. As before $h_{n} \rightarrow h \in A_{0}(\Delta)$ and $f \vee(-f) \leqq h$. Thus $0 \leqq h \in V$ and

$$
\|h\|=\|h\|_{u} \leqq \sum_{n=1}^{\infty}\left\|h_{n+1}-h_{n}\right\|_{u}+\left\|h_{1}\right\|_{u}<\|f\|+\frac{\varepsilon}{2}+\sum_{n=2}^{\infty} \frac{\varepsilon}{2^{n}}=\|f\|+\varepsilon .
$$

Together with Lemma 2.6 this proves the regularity of $V$.

4. Some special cases. We define an $R$-space $V$ as a regular ordered Banach space with the Riesz separation property. If $V$ is an ordered Banach space we say it is of type $M$ if for any $x, y \geqq 0$ we can find $z \geqq x, y$ with

$$
\|z\| \leqq \max \{\|x\|,\|y\|\}
$$

and of type $L$ if for any $x, y \geqq 0$, we have

$$
\|x+y\|=\|x\|+\|y\| \text {. }
$$

LEMMA 4.1. If $V$ is an $R$-space of type $L$ then it is a Banach lattice.

See [2].

LEMMA 4.2. If $V$ is an $R$-space of type $M$ then $V^{*}$ is a Banach lattice of type $L$.

The proof of this is trivial.

We can now give an immediate proof of a theorem of Dixmier and Kakutani [8], [14].

Theorem 4.3. Let $V$ be an ordered Banach space. Then $V$ is an L-type Banach lattice if and only if $V^{*}$ is an M-type Banach lattice. 
If $V$ is an $L$-type Banach lattice, it is an easy corollary of Lemma 2.3 that $V^{*}$ is an $M$-type Banach lattice. If $V^{*}$ is an $M$-type Banach lattice then $V$ is an $R$-space and $V^{* *}$ is an $L$-type Banach lattice by Lemma 4.2. As $V$ is canonically embedded in $V^{* *}$, so $V$ is of type $L$ and is an $L$-type Banach lattice.

THEOREM 4.4. Let $V$ be an ordered Banach space. Then $V$ is an $R$-space of type $M$ if and only if $V^{*}$ is a Banach lattice of type $L$.

For the proof see [6]. Such spaces $V$ are referred to as simplex spaces in [11]. We show how this theory is related to Choquet boundary theory, as expounded in [3], [9] and [17]. Let $V$ be an ordered Banach space with a distinguished order unit $e$ such that for all $x \in V$ we have

$$
\|x\|=\inf \{\alpha:-\alpha e \leqq x \leqq \alpha e\} .
$$

Then $V$ is regular and if

$$
\Omega=\left\{\phi \in V^{*}: \phi \geqq 0 \cdot \phi(e)=1\right\},
$$

then $\Omega$ is a weak*-compact base for the cone $C^{*}$ in the sense of [10]. $V$ is isometrically and order isomorphic with $A_{0}(\Delta)$ and with $A(\Omega)$, the space of continuous affine functionals on $\Omega$. The conical measures can be identified with the regular Borel measures on $\Omega . \Omega$ is called a simplex [4] if $C^{*}$ is lattice-ordered, and it is shown in [9], [15] that this occurs if and only if $A(\Omega)$ has the Riesz separation property.

5. The ideals in an $R$-space. If $V$ is an ordered Banach space, an order ideal $I$ in $V$ is a subspace such that if $0 \leqq x \leqq y \in I$, then $x \in I$. An ideal is defined as a positively generated order ideal. We now investigate the properties of the closed ideals of an $R$-space. These generalise the results on the closed ideals of a simplex space in [11], and provide direct proofs for those theorems.

LEMMA 5.1. Let $I$ be a closed ideal in an $R$-space $V$. Then with the restriction norm and ordering $I$ is an $R$-space.

The only part not immediate is the second half of the regularity condition. Let $x \in I$ and let $z \in I, z \geqq x,-x$. Let $y \in V$ with $y \geqq x,-x$ and $\|y\|<\|x\|+\varepsilon$. Then by the Riesz separation property there is some $\omega \in V$ with $x,-x \leqq \omega \leqq y, z$. As $0 \leqq \omega \leqq z \in I$ so $\omega \in I$ and as $0 \leqq \omega \leqq y$ so $\|\omega\| \leqq\|y\|<\|x\|+\varepsilon$. Thus $I$ is indeed regular.

LEMMA 5.2. Let $I, J$ be closed ideals in an R-space $V$. Then $I \cap J$ is a closed ideal.

We need only verify that $I \cap J$ is positively generated. Let $x \in I \cap J$ and let $y \in I, z \in J$ be such that $x,-x \leqq y$ and $x,-x \leqq z$. Then we can find $\omega \in V$ such that $x,-x \leqq \omega \leqq y, z$. As $0 \leqq \omega \leqq y$ so $\omega \in I$ and as $0 \leqq \omega \leqq z$ so $\omega \in J$. This proves the lemma. 
THEOREM 5.3. Let $I, J$ be closed ideals in an R-space $V$. Then $I+J$ is a closed ideal.

We first show that $I+J$ is positively generated. Let $x=i+j$ where $i \in I$ and $j \in J$. Then let $i_{1} \in I$ and $j_{1} \in J$ be such that $i,-i \leqq i_{1}$ and $j,-j \leqq j_{1}$. If $x_{1}=i_{1}+j_{1}$ then $x_{1} \geqq 0$ and $x,-x \leqq x_{1}$.

If in particular in the above $x \geqq 0$ then by the Riesz separation property as $0 \leqq x \leqq i_{1}+j_{1}$ we can find $0 \leqq i_{2} \leqq i_{1} \in I$ and $0 \leqq j_{2} \leqq j_{1} \in I$ such that $x=i_{2}+j_{2}$. Thus the positive cone of $I+J$ is the sum of the positive cones of $I$ and $J$. It is now immediate that $I+J$ is an order ideal. As in Theorem 5.1 we see that it is regular.

We now have only to show that $I+J$ is a closed subspace of $V$. Suppose $\varepsilon>0$ is given. Let $z \in I+J$ and let $z,-z \leqq i+j$ where $0 \leqq i \in I, 0 \leqq j \in J$ and $\|i+j\|$ $<\|z\|+\varepsilon$. Now observe that $z-j,-i \leqq i, z+j$ so by the Riesz separation property if we let $x \in V$ satisfy

$$
z-j,-i \leqq x \leqq i, z+j
$$

and put $y=z-x$ we have

$$
-i \leqq x \leqq i \text { and }-j \leqq y \leqq j
$$

so that $x \in I$ and $y \in J$. We also have

$$
\|x\| \leqq\|i\| \leqq\|i+j\|<\|z\|+\varepsilon \text { and }\|y\| \leqq\|j\| \leqq\|j+i\|<\|z\|+\varepsilon .
$$

Now let $z_{n} \in I+J$ and $\sum\left\|z_{n}\right\|<\infty$. Let $z_{n}=x_{n}+y_{n}$ where $x_{n} \in I,\left\|x_{n}\right\|<2\left\|z_{n}\right\|$ and $y_{n} \in J,\left\|y_{n}\right\|<2\left\|z_{n}\right\|$. Then we see that $\sum x_{n} \rightarrow x \in I$ and $\sum y_{n} \rightarrow y \in J$, so that $\sum z_{n} \rightarrow x+y \in I+J$. This proves that $I+J$ is closed.

THEOREM 5.4. Let I be a closed ideal in an R-space $V$ and let V/I be given the quotient norm and the positive cone which is the image of the positive cone of $V$. Then $V / I$ is an $R$-space.

Let $\widetilde{C}$ be the positive cone in $V / I$ so that $\widetilde{C}=\pi C$ where $\pi: V \rightarrow V / I$ is the quotient map. Then $\tilde{C} \cap(-\tilde{C})=\{0\}$. For let $x, y \in C$ and $\pi x=-\pi y$. Then $\pi(x+y)=0$ so that $x+y \in I$. Then $x, y \in I$ so that $\pi x=\pi y=0$.

It is clear that $V / I$ is a positively generated partially ordered space, but we do not yet show that its positive cone is closed.

Suppose $0 \leqq \tilde{y} \in V / I$. For some $x \in V$ we have $\pi x=\tilde{y}$ and $\|x\|<\|\tilde{y}\|+\varepsilon / 2$. There is also some $z \in V$ with $z \geqq 0$ and $\pi z=\tilde{y}$. As $I$ is positively generated there is some $v \in V$ such that $v \geqq x, z$ and $\pi v=\tilde{y}$. Also as $V$ is regular there is some $w \in V$ such that $w \geqq x, 0$ and $\|w\|<\|x\|+\varepsilon / 2$. If now $y \in V$ is chosen so that $0, x \leqq y \leqq w, v$ then $0 \leqq y \in V, \pi y=\tilde{y}$ and $\|y\|<\|\tilde{y}\|+\varepsilon$.

Suppose $0 \leqq \tilde{y} \leqq \tilde{z} \in V / I$ and

$$
\begin{aligned}
& 0 \leqq a \in V \text { has } \pi a=\tilde{y} \\
& 0 \leqq b \in V \text { has } \pi b=\tilde{z}-\tilde{y}, \\
& 0 \leqq c \in V \text { has } \pi c=\tilde{z} \text { and }\|c\|<\|\tilde{z}\|+\varepsilon .
\end{aligned}
$$


Then $\pi(a+b)=\pi c=\tilde{z}$. As $I$ is an ideal we can find $z \in V$ such that $0 \leqq z \leqq a+b, c$ and $\pi z=\tilde{z}$. By the Riesz decomposition property we can find $y, x \in V$ such that $0 \leqq y \leqq a$ and $0 \leqq x \leqq b$ and $x+y=z$. Then $0 \leqq \pi y \leqq \tilde{y}$ and $0 \leqq \pi x \leqq \tilde{z}-\tilde{y}$ and $\pi y+\pi x=\tilde{z}$. Thus $\pi y=\tilde{y}$. We now have $0 \leqq y \leqq z, \pi y=\tilde{y}, \pi z=\tilde{z}$ and $\|z\| \leqq\|c\|$ $<\|\tilde{z}\|+\varepsilon$. We immediately obtain

$$
\|\tilde{y}\| \leqq\|y\| \leqq\|z\|<\|\tilde{z}\|+\varepsilon
$$

and, as $\varepsilon>0$ is arbitrary, so $\|\tilde{y}\| \leqq\|\tilde{z}\|$.

Suppose $-\tilde{z} \leqq \tilde{y} \leqq \tilde{z} \in V / I$. Then $0 \leqq \tilde{y}+\tilde{z} \leqq 2 \tilde{z}$. We can from the previous paragraph find $0 \leqq u \leqq v \in V$ with $\pi u=\tilde{y}+\tilde{z}, \pi v=2 \tilde{z}$ and $\|v\|<2\|\tilde{z}\|+\varepsilon$. Then $-v / 2$ $\leqq u-v / 2 \leqq v / 2$ and $\pi(u-v / 2)=\tilde{y}$. Thus $\|\tilde{y}\| \leqq\|u-v / 2\| \leqq\|v / 2\|<\|\tilde{z}\|+\varepsilon / 2$. Thus $\|\tilde{y}\| \leqq\|\tilde{z}\|$, and we have shown that $V / I$ is a regular partially ordered Banach space.

We can now show that the positive cone in $V / I$ is closed. Let $\tilde{x}_{n} \in V / I$ where $\sum\left\|\tilde{x}_{n}\right\|<\infty$ and $\sum_{r=1}^{n} \tilde{x}_{r} \geqq 0$ for all $n$. Let $\tilde{x}_{n}=\tilde{a}_{n}-\tilde{b}_{n}$ where $\tilde{a}_{n} \geqq 0, \tilde{b}_{n} \geqq 0$ and $\sum\left\|\tilde{a}_{r}\right\|<\infty, \sum\left\|\tilde{b}_{r}\right\|<\infty$. We have $\sum_{r=1}^{n} \tilde{a}_{r} \geqq \sum_{r=1}^{n} \tilde{b}_{r}$. Now let $0 \leqq a_{n} \in V$ where $\pi a_{n}=\tilde{a}_{n}$ and $\sum\left\|a_{n}\right\|<\infty$. $\sum a_{n}$ converges in $V$ to a limit $a \geqq 0$. We now construct $0 \leqq b_{n} \in V$ such that $\sum_{r=1}^{n} b_{r} \leqq \sum_{r=1}^{n} a_{n}$ and $\left\|b_{n}\right\|<2\left\|\tilde{b}_{n}\right\|$. Suppose $b_{1}, \ldots, b_{n-1}$ have been so constructed. As $\tilde{b}_{n} \geqq 0$ so we can find $0 \leqq c_{n} \in V$ with $\pi c_{n}=\tilde{b}_{n}$ and $\left\|c_{n}\right\|$ $<2\left\|b_{n}\right\|$. As

$$
\tilde{b}_{n+1} \leqq \sum_{r=1}^{n} \tilde{a}_{r}-\sum_{r=1}^{n-1} \tilde{b}_{r}
$$

so we can find $d_{n} \in V$ with $\pi d_{n}=\tilde{b}_{n}$ and

$$
d_{n} \leqq \sum_{r=1}^{n} a_{r}-\sum_{r=1}^{n-1} b_{r}=k_{n}
$$

We can now find $e_{n} \in V$ with $e_{n} \leqq c_{n}, d_{n}$ and $\pi e_{n}=\tilde{b}_{n}$. As $0, e_{n} \leqq c_{n}, k_{n}$, so we can find $b_{n} \in V$ with $0, e_{n} \leqq b_{n} \leqq c_{n}, k_{n}$. Then $0 \leqq b_{n},\left\|b_{n}\right\| \leqq\left\|c_{n}\right\|<2\left\|\tilde{b}_{n}\right\|, \pi b_{n}=\tilde{b}_{n}$ and

$$
\sum_{r=1}^{n} b_{r} \leqq \sum_{r=1}^{n} a_{r} \leqq a \text {. }
$$

It is now clear that $\sum_{r=1}^{n} b_{r} \rightarrow b \leqq a$. Then $\sum_{r=1}^{n}\left(a_{r}-b_{r}\right) \rightarrow a-b \geqq 0$. Thus $\sum_{r=1}^{n} \tilde{x}_{r} \rightarrow x=\pi(a-b) \geqq 0$, and we have shown $V / I$ has a closed cone.

Finally we show that $V / I$ has the Riesz separation property. Suppose $\tilde{a}, \tilde{b} \leqq \tilde{c}$, $\tilde{d}$ and let $\pi a=\tilde{a}, \pi b=\tilde{b}$. We can find $c, d \geqq a, b$ with $\pi c=\tilde{c}$ and $\pi d=\tilde{d}$. By the Riesz separation property for $V$ we can find $e \in V$ with $a, b \leqq e \leqq c, d$ and if $\tilde{e}=\pi e$ we have $\tilde{a}, \tilde{b} \leqq \tilde{e} \leqq \tilde{c}, \tilde{d}$. This concludes the proof of the theorem.

Another way of investigating closed ideals, pursued for simplex spaces in [11], is to characterise their annihilators in the dual space.

Returning to the notation of $\S 2$, we define a conical face in $\Delta$ as a closed convex set $F$ with $0 \in F$ such that if $x, y \in \Delta$ and $\alpha x+\beta y \in \Delta$ for any $\alpha>0, \beta>0$ then $x, y \in F$. 
THEOREM 5.5. Let $V$ be an R-space, $V^{*}$ its dual and $\Delta=\left\{\phi \in V^{*}: \phi \geqq 0\right.$ and $\|\phi\| \leqq 1\}$. Then the maps $I \rightarrow I^{0} \rightarrow I^{0} \cap \Delta$ determine a one-one correspondence between the set of all closed ideals $I$ in $V$, the set of all weak*-closed lattice ideals in $V^{*}$, and the set of all conical faces in $\Delta$. I may naturally be identified with the space of all continuous affine functionals $f$ on $\Delta$ with $f \mid I^{0} \cap \Delta=0$.

It is elementary to show that if $I$ is a closed ideal then $I^{0}$ is a weak*-closed lattice ideal and $F=I^{0} \cap \Delta$ is a conical face. Now let $F$ be a conical face. Let $J$ be the subspace of $V^{*}$ generated by $\Delta$. Then $J$ is obviously an ideal and it meets the unit ball $B$ in a weak*-closed set. Specifically,

$$
J \cap B=(1+\varepsilon)(\Delta-\Delta) \cap B .
$$

By a well-known theorem on Banach spaces [18] it follows that $J$ is a weak*-closed subspace. $J$ is a lattice ideal with $J \cap \Delta=F$. Let $I_{1}$ be defined as the subspace of $V$ such that $f \in I$ and $\phi \in F$ implies $(f, \phi)=0$. Then $I_{1}={ }^{0} J$ is a closed subspace of $V=A^{0}(\Delta) . I_{1}$ determines and is determined by $\Delta$, and if $\Delta$ is derived from a closed ideal $I$ then $I_{1}=I$.

All we need to show is that $I_{1}$ is always an ideal. It is clearly an order ideal. Suppose $f \in I_{1}$. Let $g$ be the function $g: \Delta \rightarrow(-\infty, \infty]$ defined by

$$
\begin{aligned}
g(x) & =0 & & \text { if } x \in F, \\
& =\infty & & \text { if } x \notin F .
\end{aligned}
$$

Then $g$ is l.s.c. affine and $f,-f \leqq g$. If we can find $h \in A_{0}(\Delta)$ with $f,-f \leqq h \leqq g$, then $h \mid F=0$, so we see that $I$, is positively generated and so is an ideal. The theorem is completed by the following separation theorem.

THEOREM 5.6. Let $V$ be an $R$-space and

$$
\Delta=\left\{\phi \in V^{*}: \phi \geqq 0 \cdot\|\phi\| \leqq 1\right\}
$$

If $-f_{1}, \ldots,-f_{n}, g_{1}, \ldots, g_{m}$ are l.s.c. affine functionals on $\Delta$ vanishing at the origin such that $f_{i} \leqq g_{j}$ for all $i, j$, then we can find $h \in A_{0}(\Delta)$ with $f_{i} \leqq h \leqq g_{j}$ for all $i, j$.

First observe that if $k_{1}, \ldots, k_{p}$ are u.s.c. affine functionals vanishing at the origin then $\left(k_{1} \vee \cdots \vee k_{p}\right)^{\wedge}$ defined as in Lemma 2.7 is easily shown to be u.s.c. affine. The result now follows by a simple application of the technique of forcing convergence developed in the first part of the proof of Theorem 3.1.

\section{REFERENCES}

1. T. Andô, On fundamental properties of a Banach space with a cone, Pacific J. Math. 12 (1962), 1163-1169.

2. H. Bauer, Geordnete Gruppen mit Zerlegungseigenschaft, Bayer. S.-B. Akad. Wiss. Math.Natur. K1. 21 (1958), 25-35.

3. - Konvexität in topologischen Vectorräumen, Lecture notes, Univ. of Hamburg, Hamburg, 1963/1964. 
4. G. Choquet and P.-A. Meyer, Existence et unicité des representations intégrales dans les convexes compacts quelconques, Ann. Inst. Fourier (Grenoble) 13 (1963), 139-154.

5. G. Choquet, Séminaire du théorie du potential, Faculté des Sciences de Paris, fasc. 2 (1961/1962), 12.01-12.15.

6. E. B. Davies, On the Banach duals of certain spaces with the Riesz decomposition property, Quart. J. Math. Oxford Ser. (2) 18 (1967), 109-111.

7. M. M. Day, Normed linear spaces, Springer-Verlag, Berlin, 1958.

8. J. Dixmier, Sur certains espaces considérés par M. H. Stone, Summa Brasil. Math. 2 (1951), 151-182.

9. D. A. Edwards, Séparations des fonctions réalles définies sur un simplexe de Choquet, C. R. Acad. Sci. Paris 261 (1965), 2798-2800.

10. - On the homeomorphic affine embedding of a locally compact cone into a Banach dual space endowed with the vague topology, Proc. London Math. Soc. 14 (1964), 399-414.

11. E. G. Effros, Structure in simplexes, Lecture notes, Aarhus Univ., Aarhus, Denmark, 1965.

12. A. J. Ellis, The duality of partially ordered normed linear spaces, J. London Math. Soc. 39 (1964), 730-744.

13. R. V. Kadison, Transformations of states in operator theory and dynamics, Topology 3 (1964), 177-198.

14. S. Kakutani, Concrete representations of abstract M-spaces, Ann. of Math. 42 (1941), 994-1024.

15. J. Lindenstrauss, Extension of compact operators, Mem. Amer. Math. Soc., No. 48, $1964,112 \mathrm{pp}$.

16. I. Namioka, Partially ordered linear topological spaces, Mem. Amer. Math. Soc., No. 24, 1957.

17. R. R. Phelps, Lectures on Choquet's theorem, Van Nostrand, New York, 1966.

18. R. P. Robertson and W. J. Robertson, Topological vector spaces, Cambridge Tracts in Math. and Math. Phys., No. 53, Cambridge Univ. Press., 1964.

\section{OXFORD UNIVERSITY, \\ OXFORD, ENGLAND}

